\title{
Center-of-mass distribution of a polymer near a repulsive wall
}

\author{
E. Eisenriegler and R. Maassen \\ Institut für Festkörperforschung, Forschungszentrum Jülich, D-52425 Jülich, Germany
}

(Received 31 July 2001; accepted 8 October 2001)

[DOI: $10.1063 / 1.1423323]$

The interaction of polymer chains with a hard wall is one of the paradigms on the way to understand colloid-polymer mixtures. $^{1,2}$ A hard wall changes the structure of polymer chains in a profound way which is reflected in the forms of the various polymer density profiles. Besides the density of all the chain segments and the densities of particular segments such as the endpoints or the midpoint, the density of the center of mass of a polymer chain is of most interest. It has been pointed out by Bolhuis, Louis, Hansen, and Meijer ${ }^{2}$ that even for ideal, random-walk like, polymers no analytic expression is available for the center-of-mass density profile.

Here we fill this gap and show that the bulk-normalized center of mass density profile of free ideal polymer chains in a half space bounded by a hard planar wall ${ }^{3}$ has the form

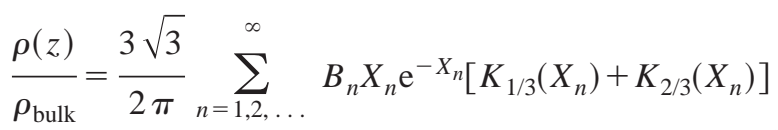

with coefficients

$$
B_{n}=\left[\int_{a_{n}}^{\infty} d Y \operatorname{Ai}(Y)\right]^{2} /\left\{\left|a_{n}\right|\left[\operatorname{Ai}^{\prime}\left(a_{n}\right)\right]^{2}\right\},
$$

and with the distance $z$ from the wall contained in

$$
X_{n}=\frac{2}{27}\left|a_{n}\right|^{3} / \zeta^{2}, \quad \zeta=z / R_{g}
$$

Here $a_{n}$ is the $n$th zero ${ }^{4}$ of the Airy function Ai, the prime in $\mathrm{Ai}^{\prime}$ denotes a derivative, $K$ is a modified Bessel function, and $R_{g}^{2}=R_{g, x}^{2}+R_{g, y}^{2}+R_{g, z}^{2}$ is the mean square radius of gyration of an ideal polymer chain in free space without a wall. As expected $\rho(z) / \rho_{\text {bulk }}$ only depends on the ratio $z / R_{g}$. For $z / R_{g} \gg 1$ the main contribution to the sum in Eq. (1) comes from $n \gg 1$. In this ${ }^{4}$ case $B_{n} \rightarrow 2 /(3 n), X_{n} \rightarrow \frac{1}{6}(\pi n / \zeta)^{2}$, the sum can be replaced by an integral, and $\rho(z) / \rho_{\text {bulk }}$ equals 1 . For $z / R_{g} \ll 1$ the first term in the sum in Eq. (1) dominates and

$$
\begin{aligned}
\frac{\rho(z)}{\rho_{\text {bulk }}} & \rightarrow \frac{1}{\sqrt{\pi}} B_{1}\left|a_{1}\right|^{3 / 2} \frac{1}{\zeta} \exp \left(-\frac{4}{27} \frac{\left|a_{1}\right|^{3}}{\zeta^{2}}\right) \\
& \rightarrow 2.85 \frac{1}{\zeta} \exp \left(-1.89 / \zeta^{2}\right) .
\end{aligned}
$$

The behavior of the center-of-mass density profile (1) for arbitrary $z / R_{g}$ is shown as the solid line in Fig. 1 (see also
Table I). The good agreement with the corresponding simulation result in Ref. 2 shows that the simulation indeed probes the universal scaling region.

For $\zeta \rightarrow 0$ the center-of-mass density profile (4) drops to zero much more rapidly than profiles for the monomer density or the density of the chain midpoint or endpoints. These display power laws ${ }^{2,5}$ proportional to $\zeta^{2}$ or $\zeta$ rather than the exponential behavior ${ }^{6}$ in Eq. (4). Note the close similarity of Eq. (4) with ${ }^{1}$ the ratio $8 \pi^{-2} \exp \left(-\pi^{2} R_{g}^{2} / D^{2}\right)$ of the partition function of a chain between two walls a small distance $D$ apart and averaged over the position of its fixed end to the partition function with fixed end in the bulk. Chain configurations contributing to a center of mass distance $z \ll R_{g}$ from a single wall to a certain extent resemble the configurations of a chain trapped between two walls with a distance $D$ of the order ${ }^{6,7}$ of $z$. It would be interesting to check whether the corresponding ${ }^{8}$ exponential behavior $\exp \left(-\right.$ const $\left./ \zeta^{1 / \nu}\right)$ for $z / R_{g} \ll 1$ appears in the center of mass distribution $\rho(z) / \rho_{\text {bulk }}$ of a single chain with excluded volume interactions between monomers. Here $\nu$ is the Flory exponent.

To derive Eq. (1) consider a single chain of $N$ segments in the space between two parallel walls at $z=0$ and $z=\mathcal{D}$. Then,

$$
\frac{\rho(z)}{\rho_{\text {bulk }}}=\lim _{\mathcal{D} \rightarrow \infty} \mathcal{D}\left\langle\delta\left(z_{\mathrm{cm}}-z\right)\right\rangle,
$$

where the angular brackets denote an average over all chain configurations. Here $z_{\mathrm{cm}}=\sum_{j=1}^{N} z_{j} / N$ is the component perpendicular to the wall of the center-of-mass of the chain with $z_{j}$ the distance from the wall of segment $j$. A Laplace transform

$$
\tau \equiv \int_{0}^{\infty} d \zeta e^{-q R_{g}^{2} z} \frac{\rho(z)}{\rho_{\text {bulk }}}=\lim _{\mathcal{D} \rightarrow \infty} \mathcal{D}\left\langle\mathrm{e}^{-q R_{g}^{2} z_{\mathrm{cm}}}\right\rangle / R_{g}
$$

with respect to the distance $z$ maps the problem onto an ideal polymer chain in a gravitational field with a strength proportional to the Laplace conjugate $q$. Following well known steps to treat an ideal chain in an external field ${ }^{9}$ one finds

$$
\tau=\sum_{n} \mathrm{e}^{-R_{g}^{2} E_{n}}\left[\int_{0}^{\infty} d \tilde{z} \psi_{n}(\tilde{z})\right]^{2} / R_{g}
$$

with a discrete set of eigenfunctions $\psi$ and eigenvalues $E$ which satisfy

$$
\left(\partial_{\tilde{z}}^{2}-q \tilde{z}+E_{n}\right) \psi_{n}(\tilde{z})=0, \quad \psi_{n}(0)=0,
$$




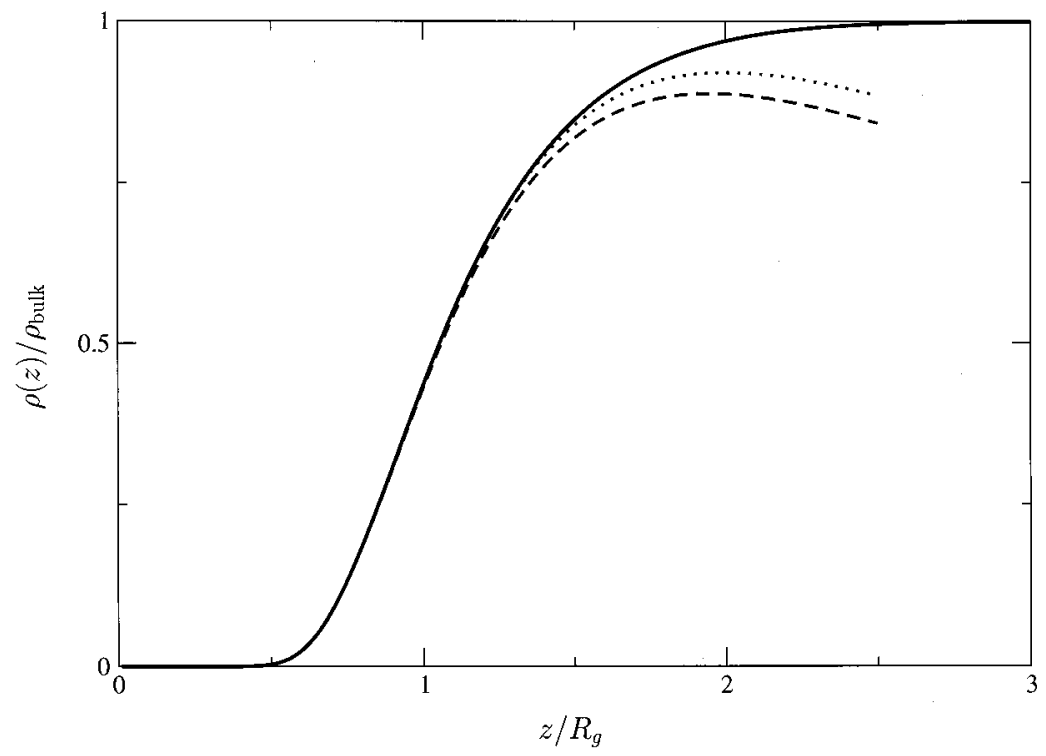

FIG. 1. Normalized center-of-mass density profile for ideal polymer chains near a hard wall as given by Eq. (1) (solid line). The dotted line shows the contribution from the "ground state" $n=1$ in Eq. (1) and the line of dashes shows the asymptotic expression given in Eq. (4).

and the orthonormality condition that $\int_{0}^{\infty} d \tilde{z} \psi_{n}(\tilde{z}) \psi_{m}(\tilde{z})$ equals one for $n=m$ and zero otherwise. The second equation in (8) is the boundary condition at the hard wall at $z$ $=0$. Explicitly this leads to ${ }^{4}$

$$
\tau \equiv \int_{0}^{\infty} d \zeta e^{-Q \zeta} \frac{\rho(z)}{\rho_{\text {bulk }}}=Q^{-1 / 3} \sum_{n} e^{-Q^{2 / 3}\left|a_{n}\right|} B_{n}\left|a_{n}\right|,
$$

where $Q=q R_{g}^{3}$. Inverting the Laplace transform ${ }^{10}$ leads to Eq. (1).

Note added in proof. We have checked that the integral $\int_{0}^{\infty} d \zeta\left[1-\rho(z) / \rho_{\text {bulk }}\right]$ with the center of mass density profile $\rho / \rho_{\text {bulk }}$ for ideal chains from Eq. (1) has the known value $2 / \sqrt{\pi}$. This integral has the same value for the different pro-

TABLE I. Some numerical values of the normalized center-of-mass density profile which follow from the analytic expression in Eq. (1).

\begin{tabular}{cc}
\hline \hline$\zeta$ & $\rho(z) / \rho_{\text {bulk }}$ \\
\hline 0.50 & 0.00294 \\
0.75 & 0.13208 \\
1.00 & 0.43420 \\
1.25 & 0.69196 \\
1.50 & 0.84699 \\
1.75 & 0.92882 \\
2.00 & 0.96907 \\
2.25 & 0.98755 \\
2.50 & 0.99538 \\
2.75 & 0.99842 \\
3.00 & 0.99951 \\
\hline \hline
\end{tabular}

files of the monomer density, the density of chain ends or midpoints, and the center of mass density and it determines the polymer induced surface free energy per unit area of the wall to be $(2 / \sqrt{\pi}) p R_{g}$. Here $p$ is the bulk osmotic pressure $k_{B} T n$ with $n$ the chain density in the bulk.

${ }^{1}$ S. Asakura and F. Oosawa, J. Chem. Phys. 22, 155 (1954).

${ }^{2}$ P. G. Bolhuis, A. A. Louis, J. P. Hansen, and E. J. Meijer, J. Chem. Phys. 114, 4296 (2001).

${ }^{3}$ For ideal polymer chains in the half plane $(d=2)$ bounded by a straight line or on the half-line $(d=1)$ bounded by a point, the center-of-mass density profiles are also given by the expressions for $d=3$ in Eqs. (1)-(3) provided we replace $\zeta$ by $\zeta=z / \sqrt{3\left(R_{g, x}^{2}+\ldots+R_{g, d}^{2}\right) / d}$. This also applies to the other ideal chain density profiles. The reason is that for $d>1$ the lateral components of ideal chain conformations factor off and cancel in numerator and denominator in expressions such as Eq. (5).

${ }^{4}$ M. Abramowitz and I. Stegun, Handbook of Mathematical Functions (Dover, New York, 1965).

${ }^{5}$ E. Eisenriegler, Polymers Near Surfaces (World Scientific, Singapore, 1993).

${ }^{6}$ For $\zeta \ll 1$ the corresponding repulsive free energy of interaction $\delta F_{\mathrm{cm}}(z)$ $=-k_{B} T \ln \left[\rho(z) / \rho_{\text {bulk }}\right]$ between the polymer center of mass and the wall is of the power law type $\propto \zeta^{-2}$ while the interaction between a fixed end $\delta F_{\text {end }}$ or a fixed midpoint $\delta F_{\text {mid }}$ of the polymer and the wall is of the much softer logarithmic type $\propto \ln (1 / \zeta)$. The qualitative difference in entropy loss is obvious since fixing the center-of-mass close to the wall restricts the polymer configurations much more than fixing an end or the midpoint. In particular $z_{\mathrm{cm}}=0$ implies that $z_{j}=0$ for all chain monomers $j$.

${ }^{7}$ The exponential contributions coincide if $D=2.3 z$.

${ }^{8}$ M. Daoud and P. G. de Gennes, J. Phys. (Paris) 38, 85 (1977)

${ }^{9}$ P. G. de Gennes, Scaling Concepts in Polymer Physics (Cornell University Press, Ithaca, 1979).

${ }^{10}$ A. Prudnikov, Yu. Brychkov, and O. Marichev, Integrals and Series (Gordon and Breach, London, 1992). 